\title{
Entzündlich-rheumatische Erkrankungen als Risikofaktoren für Osteoporose
}

\author{
Frey, D P
}

\begin{abstract}
Hintergrund Osteoporose und Fragilitätsfrakturen sind in der Allgemeinbevölkerung häufig. Im Alter von 50 Jahren haben Frauen ein Lebenszeitrisiko von ca. 50 \%, eine Fragilitätsfraktur zu erleiden, bei Männern beträgt dieses etwa $20 \%$. Verschiedene Faktoren können dieses Risiko erhöhen, darunter entzündlich-rheumatische Erkrankungen. Fragestellung und Methode Aufgrund der vorhandenen Literatur wird eine Übersicht über den Einfluss wichtiger entzündlich-rheumatischer Erkrankungen auf die Entwicklung einer Osteoporose und osteoporotischer Frakturen gegeben. Ergebnisse Am besten belegt ist die erhöhte Prävalenz der Osteoporose und der Fragilitätsfrakturen für die rheumatoide Arthritis und die ankylosierende Spondylitis. Für andere entzündlich-rheumatische Erkrankungen ist die Literatur spärlich. Da Untersuchungen jedoch zeigen, dass pro-inflammatorische Zytokine eine wichtige Rolle im Knochenmetabolismus spielen, ist davon auszugehen, dass entzündliche Erkrankungen ein erhöhtes Risiko für Osteoporose und Fragilitätsfrakturen darstellen. Kompliziert wird die Situation zusätzlich durch die oft vorliegenden Komorbiditäten, welche den Knochen unabhängig von der Entzündung beeinflussen können, sowie durch medikamentöse Therapien, insbesondere Steroide. Schlussfolgerung Bei Vorliegen einer entzündlich-rheumatischen Erkrankung sollte an das Vorliegen einer Osteoporose gedacht und allenfalls entsprechende Abklärungen durchgeführt werden. Insbesondere gilt dies für die rheumatoide Arthritis und die ankylosierende Spondylitis sowie für alle Erkrankungen, die seit mehr als 3 Monaten mit systemischen Steroiden behandelt werden. = Background Osteoporosis and fragility fractures are well-known diseases in the general population. At 50 years of age, there is a remaining lifetime risk of approximately $50 \%$ to suffer a fragility fracture for women and $20 \%$ for men. Several factors may increase this risk, in particular inflammatory rheumatic diseases. Objectives and methods Based on the existing literature, an overview is given on the influence of important inflammatory rheumatic diseases on the development of osteoporosis and osteoporotic fractures. Results The high prevalence of osteoporosis and fragility fractures is well known for rheumatoid arthritis and ankylosing spondylitis, whereas for other inflammatory rheumatic diseases only little information is available in the medical literature. However, research data indicate that pro-inflammatory cytokines play an important role in bone metabolism. Thus, all inflammatory diseases are likely to increase the risk of osteoporosis and fragility fractures. In addition, comorbidities and drug therapies, especially glucocorticoids, often result in bone deterioration independently from the inflammatory process. Conclusion In all patients with inflammatory rheumatic diseases, the risk of osteoporosis should be evaluated and screening performed if there are any risk factors. In particular, this holds for patients with rheumatoid arthritis, ankylosing spondylitis, and for patients treated with systemic glucocorticoids for more than 3 months.
\end{abstract}

DOI: https://doi.org/10.1007/s10304-014-0646-y

Posted at the Zurich Open Repository and Archive, University of Zurich

ZORA URL: https://doi.org/10.5167/uzh-207585

Journal Article

Published Version 
Originally published at:

Frey, D P (2015). Entzündlich-rheumatische Erkrankungen als Risikofaktoren für Osteoporose. Gynäkologische Endokrinologie, 13(1):28-32.

DOI: https://doi.org/10.1007/s10304-014-0646-y 
Gynäkologische Endokrinologie 2015 · 13:28-32 DOI 10.1007/s10304-014-0646-y

Online publiziert: 6. Dezember 2014

c) Springer-Verlag Berlin Heidelberg 2014

\section{Redaktion}

M. Birkhäuser, Basel

T. Strowitzki, Heidelberg

D.P. Frey

OsteoporoseZentrum, Klinik für Rheumatologie, UniversitätsSpital Zürich

\section{Entzündlich-rheumatische Erkrankungen als Risikofaktoren für Osteoporose}

rheumatische Erkrankung per se zu lokalen oder systemischen Veränderungen der Knochen führen (Erosionen, Spondylophyten, Osteoporose etc.), andererseits können Begleitumstände und medikamentöse Therapien rheumatischer Erkrankungen eine Osteoporose und ein erhöhtes Frakturrisiko zur Folge haben (Inaktivität, erhöhtes Sturzrisiko, Mangelernährung, Untergewicht, Steroidtherapie etc.). Es gilt deshalb, bei jeder entzündlich-rheumatischen Erkrankung auch an die Mitbeteiligung der Knochen zu denken; umgekehrt sollte bei einer Osteoporose auch eine zugrunde liegende rheumatische Erkrankung als sekundäre Ursache ausgeschlossen werden. wieder zu erneuern, können nere Defekte repariert und die Mikroarchitektur erhalten werden. Im Alter überwiegt schließlich der Knochenabbau, so dass es unweigerlich zu einer Negativbilanz und damit zum Knochenverlust kommt.

Verschiedene Faktoren können in dieses Gleichgewicht von Auf- und Abbau eingreifen und es zugunsten der Resorption verändern. Dazu gehören unveränderbare Faktoren wie der genetische Hintergrund, Alter, Geschlecht und Erkrankungen, aber auch beeinflussbare Faktoren wie beispielsweise der Lebensstil (Nikotin- und Alkoholabusus, Ernährung) und Medikamente (Steroide, Antiepileptika etc.).

\section{Entzündung und Knochenstoffwechsel}

Inflammatorische Erkrankungen aus dem rheumatischen Formenkreis gehen oft mit einer Veränderung des Knochenstoffwechsels einher. Einerseits kann eine
> Verschiedene Studien haben gezeigt, dass entzündliche Vorgänge den Knochenverlust begünstigen können.

So fand sich in einer Studie von Schett et al. (Bruneck-Studie) ein deutlicher $\mathrm{Zu}$ sammenhang zwischen erhöhtem highsensitivity CRP (hs-CRP) und dem Frakturrisiko in einer allgemeinen Population ohne spezifisch rheumatische Erkrankungen. Personen mit einem hs-CRP in der obersten Tertile (hs-CRP >3,0 mg/l) hatten ein bis zu 7,8-fach erhöhtes Frakturrisiko im Vergleich zu Personen mit einem hs-CRP im Bereich der tiefsten Tertile (hs-CRP <1,0 mg/l; [22]). Dies konnte in einer amerikanischen Studie bestätigt werden: Personen in der höchsten Quartile des gemessenen hs-CRP hatten ein um 34\% erhöhtes Risiko für Frakturen gegenüber Personen in der tiefsten Quartile. Die Studie zeigte außerdem, dass das Frakturrisiko mit dem Vorhandensein weiterer inflammatorischer Zytokine bzw. deren löslicher Rezeptoren (TNF- $\alpha$, TNF$\alpha$-sRI, TNF- $\alpha$-sRII, IL-6, IL-6sR, IL-2-sR) weiter anstieg $[4,19]$.

In den letzten Jahren wurde vermehrt auch der direkte Zusammenhang zwischen Zytokinen und dem Knochenstoffwechsel untersucht. So fördern die pro-inflammatorische Zytokine TNF, IL-1, IL-6, IL-7, IL-17 und PGE2 die Bildung von Osteoklasten und damit auch die Knochenresorption über die vermehrte Expression von RANKL und MCSF. TNF vermindert zudem die Knochenformation, indem DKK-1 und Sklerostin aktiviert werden, die zur Hemmung von Wnt führen, das für die Reifung und Aktivierung der Osteoblasten verantwortlich ist [23].

Aus diesen Untersuchungen folgt die Erkenntnis, dass die Verminderung der inflammatorischen Vorgänge einen wesentlichen Punkt zur Prophylaxe von Osteoporose bei entzündlichen Erkrankungen darstellt.

\section{Rheumatoide Arthritis}

Die rheumatoide Arthritis (RA) ist einerseits durch lokalisierte, andererseits durch generalisierte Knochenveränderungen gekennzeichnet. Ein frühes radiologisches Zeichen ist die gelenksnahe Osteoporose. Im späteren Verlauf kann es zu erosiven Veränderungen der Gelenke und einer generalisierten Osteoporose kommen. Während die lokalisierten Schädigungen des Knochens im Bereich der Gelenke durch die entzündlichen Vorgänge der Synovia bedingt sind, liegen der generalisierten Osteoporose meist mehrere Ursachen zugrunde: neben der Entzündungsaktivität spielen verminderte Mobilität, Steroidtherapie und allenfalls Vitamin-D-Man- 
gel eine Rolle, dazu kommt oft ein erhöhtes Sturzrisiko und eine verminderte Muskelkraft, welche zum erhöhten Frakturrisiko beitragen $[5,9,10]$.

Die Prävalenz der Osteoporose/Osteopenie bei Patientinnen mit RA wird in der Literatur mit $20-40 \%$ angegeben. Bei Männern gibt es keine verlässlichen Zahlen; die Prävalenz wird jedoch wahrscheinlich unterschätzt. Eine Untersuchung aus Deutschland 2011 ergab bei Frauen mit RA (alle Altersgruppen) eine Prävalenz der Osteoporose von 22\% (Männer 20\%) und der Osteopenie von $49 \%$ (Männer 50\%). Nur gerade 30\% aller Patientinnen und Patienten mit RA hatten eine normale Knochendichte [11].

Eine Kohortenstudie mit $150 \mathrm{RA}-\mathrm{Pa}$ tienten in Zentren der Niederlande, Norwegen und Großbritannien zeigte eine Inzidenz für nichtvertebrale Frakturen von 3,2/100 Patienten/Jahr und von vertebralen Frakturen von 3,7/100 Patienten/ Jahr. Die Patienten waren teilweise bereits unter antiosteoporotischer Therapie [26].

Studien in Populationen ohne RA ergaben dagegen Inzidenzen für vertebrale Frakturen zwischen 0,8 und 1,07/100 Patienten/Jahr. Eine neuere australische Studie ergab bei einer Kohorte aus Südaustralien eine alterskorrigierte Frakturrate von 1,9\% innerhalb von 3 Jahren verglichen zu $1,2 \%$ bei Patientinnen ohne RA. Patientinnen mit RA haben somit ein deutlich erhöhtes Frakturrisiko gegenüber Patientinnen ohne RA [2].

Gemäß der aktuell noch gültigen Leitlinie 2009 des Dachverbandes Osteologie soll eine Osteoporosediagnostik bei rheumatoider Arthritis bei Frauen über 60 Jahren und Männern ab 70 Jahren durchgeführt werden. Im Entwurf der neuen Leitlinie 2014 wird eine Abklärung hingegen schon bei allen postmenopausalen $\mathrm{Pa}$ tientinnen unabhängig vom Alter sowie bei Männern über 60 Jahren empfohlen. Bei jüngeren Patientinnen und Patienten ist die Basisabklärung eine Einzelfallentscheidung. Bei gleichzeitiger Behandlung mit Steroiden und/oder anderen Risikofaktoren ist jedoch sicher eine frühe $\mathrm{Ab}$ klärung angezeigt.

Die Basisdiagnostik beinhaltet neben einer genauen Anamnese (evtl. andere anamnestische Risikofaktoren) und klinischen Untersuchung (mögliche Hinweise für bestehende Wirbelfrakturen oder andere sekundäre Ursachen der Osteoporose) eine DXA-Messung (Knochendichtemessung) sowie ein Labor zum Ausschluss von anderen Erkrankungen, welche eine Osteoporose verursachen können. Bei klinischen Hinweisen auf eine Wirbelfraktur sollte außerdem eine konventionell-radiologische Abklärung der BWS (Brustwirbelsäule) und LWS (Lendenwirbelsäule) durchgeführt werden [12].

\section{\) Bei einer rheumatoiden Arthritis muss Vitamin D substituiert werden}

Für alle Patientinnen mit RA gilt, dass eine optimale Kalzium- und VitaminD-Zufuhr erfolgen soll -mindestens 1000 mg Kalzium sowie 800-1000 IU Vitamin D täglich als Basistherapie, wobei die Kalziumzufuhr auch über eine genügende Aufnahme mit der Nahrung erfolgen kann. Da hingegen allein durch die Ernährung meist kein genügender Vitamin-D-Spiegel erreicht werden kann, ist eine entsprechende Supplementation notwendig. Bei Patientinnen mit Osteopenie/Osteoporose kann die Bestimmung des 25-OH-Vitamin-D-Spiegels zur Festlegung der Supplementationsdosis helfen.

Wie bei Nicht-RA-Patientinnen ist die Beratung bezüglich Lifestyle (Ernährung, Noxen, Bewegung) ein Grundpfeiler der Osteoporosebehandlung. Da die Inflammation einen wesentlichen Faktor für die Entwicklung einer Osteoporose darstellt, ist die optimale Behandlung der Grundkrankheit ein entscheidender Punkt des Osteoporosemanagements.

Eine spezifische Osteoporosetherapie ist je nach Komedikation, anderen Begleiterkrankungen, Compliance und möglichen Nebenwirkungen einzuleiten. Während bei postmenopausalen Frauen mit RA und Osteoporose grundsätzlich alle Antiresorptiva gegeben werden können (Zulassung, Kostenübernahme durch die Krankenkasse), ist dies bei prämenopausalen Frauen und bei Männern oftmals schwieriger, da im Einzelfall eine Kostengutsprache eingeholt werden muss. Die Therapie muss deshalb maßgeschneidert erfolgen.

\section{Spondyloarthritiden}

Unter Spondyloarthritiden werden eine Gruppe überlappender Erkrankungen zusammengefasst, deren gemeinsamer Nenner im Wesentlichen der entzündliche Rückenschmerz mit Befall des axialen Skeletts ist. Dazu kommen je nach Art der Erkrankung der Befall von Gelenken, Enthesitiden sowie nichtskelettale Manifestationen. $\mathrm{Zu}$ den Spondyloarthritiden gehören die klassische Spondylitis ankylosans (AS; M. Bechterew), aber auch die Psoriasisarthritis (PsA), die reaktiven Arthritiden sowie die enteropathischen Arthritiden.

Da sich diese Erkrankungen trotz gemeinsamer klinischer, laborchemischer und radiologischer Kriterien doch wesentlich unterscheiden, ist es nicht erstaunlich, dass auch bezüglich der osteologischen Problematik Differenzen vorhanden sind.

\section{Spondylitis ankylosans}

Bei der AS findet sich im Gegensatz zur rheumatoiden Arthritis neben dem Knochenverlust auch eine Knochenproliferation im Sinne von Syndesmophyten. Die durch den Befall der Wirbelsäule bedingten Schmerzen und allenfalls die Mobilitätseinschränkung der Patienten begünstigen das Sturzrisiko und damit die Entstehung von Frakturen. Unabhängig davon haben die Patienten auch oft eine Osteoporose, weshalb insgesamt das Frakturrisiko bei Patienten mit AS deutlich erhöht ist.

\section{) Die Prävalenz der \\ Osteoporose wird unterschätzt}

In der Literatur wird übereinstimmend eine verminderte Knochendichte der LWS bereits in frühen Krankheitsstadien angegeben $[8,14,24]$. Im Spätstadium der Erkrankung mit ausgeprägten Syndesmophyten ist die mittels DXATechnik gemessene Knochendichte jedoch oft falsch-hoch, weshalb die Prävalenz der Osteoporose sicher unterschätzt wird. Eine Möglichkeit, die Schwierigkeit falsch-hoher Knochendichtewerte der LWS zu vermeiden, ist die Messung der 
lateralen LWS, da dadurch die ventralen Syndesmophyten sowie mögliche Knochenneubildungen im Bereich der Fazettengelenke nicht mit abgebildet werden. Diese Technik wird jedoch zur Diagnostik wegen der großen Variabilität und fehlenden Validierung nicht empfohlen [13], kann aber für die Verlaufsdokumentation eingesetzt werden. Eine weitere Möglichkeit der Knochendichtemessung besteht in der Untersuchung von LWS und Hüfte mittels quantitativer Computertomographie (QCT) sowie der peripheren Knochen (Tibia und Vorderarm) mittels hochauflösenden peripheren Computertomographien (HRpQCT). HRpQCT-Untersuchungen konnten zeigen, dass die volumetrische Knochendichte im peripheren Skelett (Tibia und Radius) tiefer war als bei gesunden Kontrollen. Die nicht nur im vorwiegend betroffenen axialen Skelett erniedrigte Knochendichte spricht für allgemein entzündliche Vorgänge als Ursache für die Osteoporose [15].

Das Frakturrisiko für vertebrale Frakturen ist deutlich erhöht, während für nichtvertebrale Frakturen die Angaben nicht einheitlich sind. Eine große niederländische Fall-Kontroll-Studie ergab ein mehr als dreifach höheres Wirbelfrakturrisiko für AS-Patienten im Vergleich zur Nicht-AS-Population während das Risiko für Vorderarm- und Hüftfrakturen nicht unterschiedlich war [27].

\section{Psoriasisarthritis}

Zu Prävalenz von Osteoporose und Fragilitätsfrakturen bei PsA existieren nur wenige Studien und die Patientenzahlen sind oft klein. Die Resultate sind widersprüchlich. Eine neuere Studie aus Spanien fand keine unterschiedlichen Knochendichtewerte zwischen PsA-Patienten und Kontrollen, während das Frakturrisiko leicht erhöht war. Insbesondere bei postmenopausalen Frauen und bei langer Krankheitsdauer gibt es Hinweise, dass das Frakturrisiko erhöht sein könnte $[6,7,20,21]$.

\section{Enteropathische Arthritiden}

Die Arthritiden aufgrund entzündlicher Darmerkrankungen (Morbus Crohn, Colitis ulcerosa) gehen ebenfalls mit einer er-

Gynäkologische Endokrinologie 2015 · 13:28-32 DOI 10.1007/s10304-014-0646-y

(c) Springer-Verlag Berlin Heidelberg 2014

\section{D.P. Frey \\ Entzündlich-rheumatische Erkrankungen als Risikofaktoren für Osteoporose}

\section{Zusammenfassung}

Hintergrund. Osteoporose und Fragilitätsfrakturen sind in der Allgemeinbevölkerung häufig. Im Alter von 50 Jahren haben Frauen ein Lebenszeitrisiko von ca. 50\%, eine Fragilitätsfraktur zu erleiden, bei Männern beträgt dieses etwa 20\%. Verschiedene Faktoren können dieses Risiko erhöhen, darunter entzündlich-rheumatische Erkrankungen.

Fragestellung und Methode. Aufgrund der vorhandenen Literatur wird eine Übersicht über den Einfluss wichtiger entzündlich-rheumatischer Erkrankungen auf die Entwicklung einer Osteoporose und osteoporotischer Frakturen gegeben.

Ergebnisse. Am besten belegt ist die erhöhte Prävalenz der Osteoporose und der Fragilitätsfrakturen für die rheumatoide Arthritis und die ankylosierende Spondylitis. Für andere entzündlich-rheumatische Erkrankungen ist die Literatur spärlich. Da Untersuchungen jedoch zeigen, dass pro-inflammatorische Zytokine eine wichtige Rolle im Knochenmetabolismus spielen, ist davon auszugehen, dass entzündliche Erkrankungen ein erhöhtes Risiko für Osteoporose und Fragilitätsfrakturen darstellen. Kompliziert wird die Situation zusätzlich durch die oft vorliegenden Komorbiditäten, welche den Knochen unabhängig von der Entzündung beeinflussen können, sowie durch medikamentöse Therapien, insbesondere Steroide.

Schlussfolgerung. Bei Vorliegen einer entzündlich-rheumatischen Erkrankung sollte an das Vorliegen einer Osteoporose gedacht und allenfalls entsprechende Abklärungen durchgeführt werden. Insbesondere gilt dies für die rheumatoide Arthritis und die ankylosierende Spondylitis sowie für alle Erkrankungen, die seit mehr als 3 Monaten mit systemischen Steroiden behandelt werden.

Schlüsselwörter

Osteoporosefrakturen $\cdot$ Knochenbrüchigkeit . Entzündungen · Rheumatoide Arthritis .

Ankylosierende Spondylitis

\section{Inflammatory rheumatic diseases as a risk for osteoporosis}

\section{Abstract}

Background. Osteoporosis and fragility fractures are well-known diseases in the general population. At 50 years of age, there is a remaining lifetime risk of approximately $50 \%$ to suffer a fragility fracture for women and $20 \%$ for men. Several factors may increase this risk, in particular inflammatory rheumatic diseases.

Objectives and methods. Based on the existing literature, an overview is given on the influence of important inflammatory rheumatic diseases on the development of osteoporosis and osteoporotic fractures.

Results. The high prevalence of osteoporosis and fragility fractures is well known for rheumatoid arthritis and ankylosing spondylitis, whereas for other inflammatory rheumatic diseases only little information is available in the medical literature. However, research data indicate that pro-inflammatory cyto- kines play an important role in bone metabolism. Thus, all inflammatory diseases are likely to increase the risk of osteoporosis and fragility fractures. In addition, comorbidities and drug therapies, especially glucocorticoids, often result in bone deterioration independently from the inflammatory process.

Conclusion. In all patients with inflammatory rheumatic diseases, the risk of osteoporosis should be evaluated and screening performed if there are any risk factors. In particular, this holds for patients with rheumatoid arthritis, ankylosing spondylitis, and for patients treated with systemic glucocorticoids for more than 3 months.

\section{Keywords}

Osteoporotic fractures · Bone fragility . Inflammation - Rheumatoid arthritis . Ankylosing spondylitis niedrigten Knochendichte und einem erhöhten Frakturrisiko einher. Beim Morbus Crohn dürfte dabei auch die Malabsorption, insbesondere von Kalzium und Vitamin D, eine Rolle spielen, während bei der Colitis ulcerosa ohne Befall des Dünndarms die Malnutrition kaum zur
Entwicklung einer Osteoporose beiträgt. Die Angaben zum Frakturrisiko variieren in der Literatur beträchtlich, je nachdem ob für begleitende Komorbiditäten korrigiert wurde (Steroidtherapie, Vitamin-DMangel etc. [25]). 


\section{Systemische Sklerose}

Spezifische Veränderungen bei systemischer Sklerose können eine Osteoporose begünstigen: chronische Entzündung, Immobilisation und allenfalls ein vermindertes Kalzium durch Gewebskalzifizierung sowie bei Frauen der frühzeitige Eintritt der Menopause. Zusätzlich kann es zu einem Vitamin-D-Mangel durch verminderte Synthesefähigkeit der Haut, Resorptionsstörungen bei Befall des Gastrointestinaltrakts und mangelnder Hydroxylierung bei Nierenfunktionsstörungen kommen. Ob Patienten mit der diffusen Form der systemischen Sklerose tiefere Knochendichtewerte aufweisen als $\mathrm{Pa}$ tienten mit der umschriebenen Form, ist umstritten. Das Frakturrisiko ist bei Patienten mit systemischer Sklerose wahrscheinlich ebenfalls erhöht, Daten dazu fehlen jedoch weitgehend $[16,18,28]$.

\section{Lupus erythematosus}

Auch der systemische Lupus erythematosus (SLE) geht mit einer deutlich verminderten Knochendichte und einem erhöhten Frakturrisiko einher. Neben der allgemeinen entzündlichen Aktivität ist bei SLE die Steroidtherapie ein wichtiger Faktor. Patienten mit Nierenbeteiligung entwickeln zudem oft einen sekundären Hyperparathyreoidismus mit entsprechenden osteologischen Folgen [1, 3, 17, 29, 30].

\section{Schlussfolgerung}

Die meisten entzündlich-rheumatischen Erkrankungen können zu einer verminderten Knochendichte bis hin zur Osteoporose führen und gehen mit einem erhöhten Frakturrisiko einher. Eine Ausnahme bildet dabei möglicherweise die PsA. Wegen der Heterogenität der Erkrankungen und den verschiedenen extraskelettalen Begleiterkrankungen sind jedoch die Ursachen, die zu einer Osteoporose führen, schwierig zu eruieren. $\mathrm{Ne}$ ben inflammatorischen Vorgängen sind es einerseits die Einschränkung der Mobilität, die vielen rheumatischen Erkrankungen inhärent ist, andererseits der Befall anderer Organe. So kann ein Hautbefall die Vitamin-D-Synthese vermindern, eine Nierenfunktionsstörung einen se- kundären Hyperparathyreoidismus verursachen und eine gastrointestinale Beteiligung zur Malabsorption führen. Zusätzlich können eine Steroidtherapie oder andere medikamentöse Therapien den Knochenmetabolismus verändern.

\section{》) Risikofaktoren für eine Osteoporose sollten reduziert werden}

Für die Praxis ist es demnach wichtig, dass im Rahmen der Betreuung von Patienten mit entzündlich-rheumatischen Erkrankungen zumindest die generellen Risikofaktoren für eine Osteoporose erfragt und - wenn möglich - reduziert werden (Nikotin- und Alkoholkonsum, Untergewicht, Ernährung etc.) und die Erkrankung bestmöglich therapiert wird, um sekundäre Organschädigungen zu vermindern. Bei einer systemischen Steroidtherapie über eine Dauer von 3 Monaten ist außerdem eine Basisabklärung indiziert. Bei Vorliegen weiterer Risikofaktoren für eine Osteoporose sollte auch ohne Steroidtherapie eine Basisdiagnostik erfolgen.

\section{Fazit für die Praxis}

- Entzündlich-rheumatische Erkrankungen gehen oft mit einem erhöhten Osteoporose- und Frakturrisiko einher.

- Die entzündliche Aktivität ist ein wichtiger Faktor für die Entwicklung einer Osteoporose.

- Der Befall weiterer Organe mit entsprechendem Funktionsverlust spielt zusätzlich zur muskuloskelettalen Manifestation eine Rolle für das Auftreten von Osteoporose und Fragilitätsfrakturen.

- Bei entzündlich-rheumatischen Erkrankungen, v. a. wenn es sich um eine RA oder Spondylitis ankylosans handelt, sollte frühzeitig an eine Osteoporose gedacht und diese entsprechend abgeklärt werden.

- Die Reduktion der Entzündungsaktivität, möglichst kurzdauernde und niedrigdosierte Steroidtherapien sowie die Behandlung von Begleiterkrankungen sind die drei wichtigsten Eckpfeiler der Therapie.

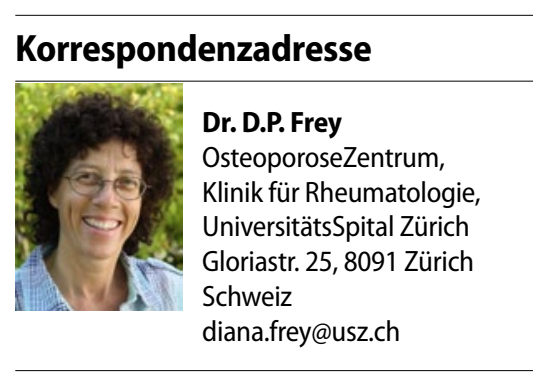

Einhaltung ethischer Richtlinien

Interessenkonflikt. D.P. Frey gibt an, dass kein Interessenkonflikt besteht.

Dieser Beitrag beinhaltet keine Studien an Menschen oder Tieren.

\section{Literatur}

1. Adachi JD, Lau A (2014) Systemic lupus erythematosus, osteoporosis, and fractures. J Rheumatol 41(10):1913-1915

2. Brennan SL, Toomey L, Kotowicz MA et al (2014) Rheumatoid arthritis and incident fracture in women: a case-control study. BMC Musculoskelet Disord 15:13

3. Bultink IE (2012) Osteoporosis and fractures in systemic lupus erythematosus. Arthritis Care Res (Hoboken) 64(1):2-8

4. Cauley JA, Danielson ME, Boudreau RM et al (2007) Inflammatory markers and incident fracture risk in older men and women: the Health Aging and Body Composition Study. J Bone Miner Res 22(7):1088-1095

5. Coulson KA, Reed G, Gilliam BE et al (2009) Factors influencing fracture risk, $T$ score, and management of osteoporosis in patients with rheumatoid arthritis in the Consortium of Rheumatology Researchers of North America (CORRONA) registry. J Clin Rheumatol 15(4):155-160

6. D'Epiro S, Marocco C, Salvi M et al (2014) Psoriasis and bone mineral density: implications for longterm patients. J Dermatol 41(9):783-787

7. Del Puente A, Esposito A, Parisi A et al (2012) Osteoporosis and psoriatic arthritis. J Rheumatol Suppl 89:36-38

8. Donnelly S, Doyle DV, Denton A et al (1994) Bone mineral density and vertebral compression fracture rates in ankylosing spondylitis. Ann Rheum Dis 53(2):117-121

9. El Maghraoui A, Rezqi A, Mounach A et al (2010) Prevalence and risk factors of vertebral fractures in women with rheumatoid arthritis using vertebral fracture assessment. Rheumatology (Oxford) 49(7):1303-1310

10. Gullick NJ, Scott DL (2011) Co-morbidities in established rheumatoid arthritis. Best Pract Res Clin Rheumatol 25(4):469-483

11. Heberlein I, Demary W, Bloching H et al (2011) Prophylaxis and treatment of osteoporosis in patients with rheumatoid arthritis (ORA study). Z Rheumatol 70(9):793-798, 800-802

12. http://www.dv-osteologie.org/dvo_leitlinien/dvoleitlinie-2009. Zugegriffen: 05. Okt. 2014

13. http://www.iscd.org/official-positions/2013-iscdofficial-positions-adult/. Zugegriffen: 05. Okt. 2014 
14. Klingberg E, Geijer M, Göthlin J et al (2012) Vertebral fractures in ankylosing spondylitis are associated with lower bone mineral density in both central and peripheral skeleton. J Rheumatol 39(10):1987-1995

15. Klingberg E, Lorentzon M, Göthlin J et al (2013) Bone microarchitecture in ankylosing spondylitis and the association with bone mineral density, fractures, and syndesmophytes. Arthritis Res Ther 15(6):R179

16. Loucks J, Pope JE (2005) Osteoporosis in scleroderma. Semin Arthritis Rheum 34(4):678-682 (Review)

17. Mak A, Lim JQ, Liu Y et al (2013) Significantly higher estimated 10-year probability of fracture in lupus patients with bone mineral density comparable to that of healthy individuals. Rheumatol Int 33(2):299-307

18. Omair MA, Pagnoux C, McDonald-Blumer H, Johnson SR (2013) Low bone density in systemic sclerosis. A systematic review. J Rheumatol 40(11):18811890

19. Pasco JA, Kotowicz MA, Henry MJ et al (2006) High-sensitivity C-reactive protein and fracture risk in elderly women. JAMA 296(11):1353-1355

20. Pedreira PG, Pinheiro MM, Szejnfeld VL (2011) Bone mineral density and body composition in postmenopausal women with psoriasis and psoriatic arthritis. Arthritis Res Ther 13(1):R16

21. Riesco M, Manzano F, Font P et al (2013) Osteoporosis in psoriatic arthritis: an assessment of densitometry and fragility fractures. Clin Rheumatol 32(12):1799-1804

22. Schett G, Kiechl S, Weger S et al (2006) High-sensitivity C-reactive protein and risk of nontraumatic fractures in the Bruneck study. Arch Intern Med 166(22):2495-2501

23. Schett G, Saag KG, Bijlsma JW (2010) From bone biology to clinical outcome: state of the art and future perspectives. Ann Rheum Dis 69(8):14151419

24. Singh HJ, Nimarpreet K, Ashima et al (2013) Study of bone mineral density in patients with ankylosing spondylitis. J Clin Diagn Res 7(12):2832-2835

25. Targownik LE, Bernstein CN, Leslie WD (2014) Risk factors and management of osteoporosis in inflammatory bowel disease. Curr Opin Gastroenterol 30(2):168-174

26. Vis $M$, Haavardsholm EA, Bøyesen P et al (2011) High incidence of vertebral and non-vertebral fractures in the OSTRA cohort study: a 5-year follow-up study in postmenopausal women with rheumatoid arthritis. Osteoporos Int 22(9):24132419

27. Vosse D, Landewé R, Heijde D van der et al (2009) Ankylosing spondylitis and the risk of fracture: results from a large primary care-based nested casecontrol study. Ann Rheum Dis 68(12):1839-1842

28. Wan YN, Zhang L, Wang YJ et al (2014) Association between systemic sclerosis and bone mineral density based on twelve observational studies. Int J Rheum Dis

29. Yee CS, Crabtree N, Skan J et al (2005) Prevalence and predictors of fragility fractures in systemic lupus erythematosus. Ann Rheum Dis 64(1):111-113

30. Zhu TY, Griffith JF, Au SK et al (2014) Bone mineral density change in systemic lupus erythematosus: A 5-year Followup Study. J Rheumatol 41(10):1990-1997
Pamela Emmerling

\section{Ärztliche Kommunikation}

Als Erstes heile mit dem Wort ...

Stuttgart: Schattauer 2014, 1. Auflage, 264 S., 8 Abb., (ISBN 978-3794529742), 29,00 EUR

Mit einem Vorwort von Frank Ulrich

Montgomery

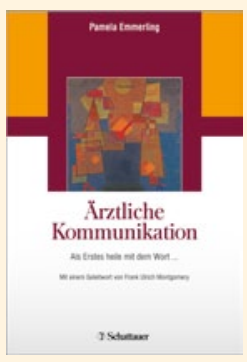

Das Buch von Frau

Pamela Emmerling

beschreibt anschau-

lich viele Hürden

eines ärztlichen Ge-

spräches, die es zu

umschiffen gilt.

In den ersten Kapiteln

werden neben den

bekannten Kommunikationsmodellen von

F. Schulz-von-Thun, Watzlawick, Mehrabian

und Rosenberg weitere Modelle aufgeführt,

die beeindruckend klar den täglichen Ablauf

in der Praxis und in jeder Art von Kommuni-

kation darlegen. Die „eigenen Impulse“ laden

jeweils zum Nachdenken, ja sogar zum Be-

arbeiten ein und veranlassen zum kritischen

Blick hinter die eigenen Kulissen eingefahrener Kommunikationsschemata.

Im Kapitel „Du Patient - andere verstehen" werden Grundausrichtungen (z.B. RiemannThomann-Modell) und "Lebensverträge " dargelegt, die häufig unbemerkt Interaktionen zwischen Gesprächspartnern aufzeigen. Die Autorin legt einfach, aber umso anschaulicher die Zusammenhänge in Beispielen dar (Dauer-Mensch, Wechsel-Mensch, DistanzMensch, Nähe-Mensch). Übersichtlich lässt sich so das Patientenverhalten zuordnen und verstehen

Die Transaktionsanalyse findet als Persön-

lichkeits- und Kommunikationskonzept

anschauliche Anwendungsfelder. Häufig

kommt es beim Lesen zum Aha-Effekt und zur

Reflexion des eigenen wie auch des fremden Verhaltens.

Im Kapitel „Ich Arzt - sich selbst verstehen" wird versucht, dem Arzt den Spiegel vorzuhalten. Bekannte, aber auch unbekannte Verhaltensweisen und Rollen werden anschaulich dargelegt. Besonders interessant und zum Nachdenken anregend ist die bekannte Darstellung des Rosenthal-Effektes sowie die Darlegung unseres Rollenverhaltens. Vielfach erlebt man große Unterschiede in Bezug auf verschiedene Situationen. Das Buch legt hier ausdrücklich dar, dass es zu den Kernkom- petenzen gehört, sich den Bedürfnissen und Erfordernissen situativ anzupassen. Letztendlich erhält man mit der Kenntnis über Modelle, Konzepte und Persönlichkeitsmerkmale die Chance, Kommunikationsdefizite zu erkennen, Maßnahmen zu ergreifen und so bessere Leistungen zu erreichen. Bessere Leistungen heißt hier vor allem weniger belastende Kommunikationsdefizite, weniger Missverständnisse, weniger Vorwürfe und weniger Zeitverluste durch unnötige Erklärungen und Korrekturen.

Auch Fragetechniken gehören zum Inhalt des Buches und werden zu einem Kernelement. Oft hat man das Gefühl, der Patient verstehe uns nicht. Wie oft fragt man dann aus Zeitgründen nicht nach. Nach Lesen des Buches erhält man die Möglichkeit, zeitnah das Problem zu erkennen und zu beheben. Wie gut, wenn jetzt die Compliance stimmt.

Entscheidend ist m.E. dass man das Buch mit all seinen umfassenden Möglichkeiten bewusst liest und im Alltag umzusetzen versucht. Es werden die Augen geöffnet. Wer sind wir, wie werden wir gesehen?

- Antreiber oder Quälgeist für Mitarbeiter und Patienten

- Partner und Garant für den Therapieerfolg

Eine sichere Kommunikation ist nicht nur im Umgang mit Patienten, Mitarbeitern, Familie und Freunden erforderlich, hilfreich erscheint sie auch in Prüfungssituationen, im heutigen Wandel der Medizinstrukturen von der Einzelpraxis zum MVZ, von kleinen, überschaubaren Krankenhäuser in Kompetenzzentren und Großkliniken.

Liest man das Buch mit offenen Augen und versucht die Übungen ehrlich durchzuführen und unter den Punkten ",die eigenen Impulse" sich mit sich und den eingefahrenen Strukturen auseinanderzusetzen, so ist das Buch ein Gewinn und eine Hilfe für alle im Job: „alte Hasen“ wie "junge Hüpfer".

S. Menke (Niebüll) 